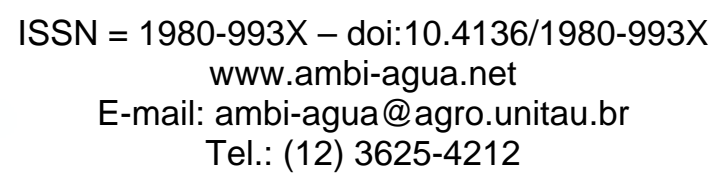

\title{
Modelagem hidrossedimentológica de bacia hidrográfica na região sudeste do Brasil, utilizando o SWAT \\ (doi:10.4136/ambi-agua.145)
}

\author{
Thatiana Aparecida Lelis; Maria Lúcia Calijuri
}

Universidade Federal de Viçosa - UFV

Email: thatianalelis@yahoo.com.br; calijuri@ufv.br

\section{RESUMO}

A avaliação quantitativa da perda de solo por erosão, da perda de água e das cargas de sedimentos que atingem os corpos d'água é fundamental para o planejamento ambiental em escala de bacia hidrográfica, contribuindo para o processo de tomada de decisão relacionado às melhores opções de manejo do solo e à manutenção da qualidade dos recursos naturais. Estimativas desses dados têm sido realizadas ao redor do mundo, com base na utilização de modelos empíricos ou conceituais. Além de uma forma econômica no levantamento das informações e na elaboração de cenários, os modelos ambientais podem contribuir para a localização de áreas críticas, fornecendo indicativos para operações emergenciais de contenção, em função da ação dos processos erosivos. Dentre esses modelos, destaca-se o SWAT (Soil and Water Assessment Tool), o qual foi aplicado na bacia do ribeirão São Bartolomeu, localizada na Zona da Mata de Minas Gerais, região Sudeste do Brasil, para identificação das zonas de maior sensibilidade à erosão em relação ao uso e tipo do solo. Paralelamente, entre 2006 e 2008, foram instaladas 10 parcelas experimentais nas culturas predominantes da bacia, o que permitiu o monitoramento do escoamento superficial e das perdas de solo sob condições de chuva natural. Os resultados de campo e das simulações mostraram a eficiência do SWAT na caracterização da produção de sedimentos e que as perdas de solo, as quais são influenciadas por fatores como umidade do solo, intensidade de precipitação, uso e tipo de solo (na região predominam os latossolos, argissolos, cambissolos e gleissolos), podem ser reduzidas significativamente pela substituição de culturas com melhor manejo. Um resultado de simulação de cenário de substituição de parte da cobertura de pastagens por eucalipto foi apresentado, o qual reduziu significativamente a perda de solo em várias regiões da bacia.

Palavras-chave: Perda de solo; erosão; modelagem ambiental.

\section{Hydrosedimentological modeling of watershed in southeast Brazil, using SWAT}

\section{ABSTRACT}

The quantitative evaluation of soil loss due to erosion, of water loss and of load sediments that reach water bodies is fundamental to the environmental planning of a watershed, contributing to the process of decision for best options for soil tillage and water quality maintenance. Estimates of these data have been accomplished throughout the world using empiric or conceptual models. Besides being economically viable in scenarios development, environmental models may contribute to the location of critical areas, leading to emergency contention operations caused by erosive processes. Among these models, we highlight the SWAT (Soil and Water Assessment Tool) which was applied in São Bartolomeu watershed, located in the Zona da Mata, Minas Gerais state, southeastern Brazil, to identify areas of greater sensitivity to erosion considering the soil type and land use. To validate the 
model, 10 experimental plots were installed in the dominant crops of the watershed between 2006 and 2008, for monitoring the runoff and soil losses under natural rainfall. Field results and simulations showed the SWAT efficiency for sediment yield and soil losses estimations, as they are influenced by factors such as soil moisture, rainfall intensity, soil type and land use (dominated by Oxisols, Ultisols, Inceptisols and Entisols). These losses can be reduced significantly by improving crops management of. A simulation scenario replacing pastures cover by Eucalyptus was introduced, which significantly reduced soil loss in many parts of the watershed.

Keywords: Soil loss; erosion; environmental modeling.

\section{INTRODUÇÃO}

O uso inadequado dos solos agrícolas vem causando a perda gradual da sua capacidade produtiva e a contaminação dos recursos hídricos por sedimentos e poluentes de origem difusa, decorrentes do processo erosivo ao qual esses solos estão sujeitos. A ação antrópica, por meio da utilização de práticas que destroem o equilíbrio das condições naturais, tem acelerado esses processos de maneira intensa, trazendo, em consequência, prejuízos ao setor agrícola e ao meio ambiente, com reflexos econômicos e sociais (Amorim, 2000; Tucci, 1998). Soma-se, ainda, a preocupação com o agravamento do processo erosivo em muitas partes do mundo, em função dos efeitos das mudanças ambientais globais (Amore et al., 2004).

Numa visão holística de preservação dos recursos naturais, a identificação dos problemas ambientais de uma área deve subsidiar a implementação de um planejamento do uso e ocupação dos solos. Todavia, para que tais práticas conservacionistas resultem em melhoria significativa e a custos aceitáveis é necessário que se proceda a uma avaliação ambiental, a fim de se obter maior precisão em relação à localização e quais delas devam ser implantadas (Prado, 2005). Nesse contexto, modelos matemáticos de estimativa de perdas de solo por erosão vêm sendo aplicados com eficiência, tanto no planejamento conservacionista (preventivo) quanto na avaliação e controle do processo erosivo de bacias hidrográficas (Machado, 2002; Christofoletti, 1999).

Dentre os modelos disponíveis para a modelagem hidrológica e hidrossedimentológica de bacias hidrográficas destaca-se o SWAT (Soil and Water Assessment Tool). O SWAT foi desenvolvido para verificar os efeitos resultantes das modificações no uso do solo sobre o escoamento superficial e subterrâneo, bem como na produção de sedimentos, tendo sido estudado e difundido amplamente pelos trabalhos de Arnold et al. (1990, 1995 e 1998), Srinivasan e Arnold (1994), Biesbrouck et al (2002), Jha et al. (2002), Gassman et al. (2003 e 2007), Neitsch et al (2005), Santhi et al. (2006), Arabi et al. (2008) e Griensven (2005). Destaca-se, também, a aplicação mundial do modelo, com resultados satisfatórios, como os obtidos por Tripathi et al. (2003) na Índia; Santhi et al. (2006), Gassman et al. (2007) e Zhang et al. (2008) nos EUA; Diez (2002), Vahos Montoya (2003) e Oñate Valdivieso e Aguilar Naranjo (2003) na America do Sul; Nunes e Pacheco (2004), Guerreiro et al. (2005), Duarte et al. (2007) e Galvan et al. (2007) na Europa; e Schuol et al. (2008) na África.

A modelagem ambiental no SWAT é realizada com uma grande quantidade de componentes hidrológicos numa bacia, de maneira abrangente, simples e realista (Muleta e Nicklow, 2005). Juntamente com os dados de crescimento das culturas, manejo e qualidade da água, as rotinas hidrológicas dentro do SWAT simulam os processos de escoamento superficial e subsuperficial, infiltração, evaporação, consumo pelas plantas, fluxo lateral, percolação e produção de sedimentos. Essas rotinas são aplicadas a todas as sub-bacias geradas pelo modelo, permitindo que diferenças nas suas características hidrológicas sejam consideradas (Minoti, 2006). Além do mais, cada sub-bacia, no SWAT, pode ser parametrizada usando uma série de Unidades de Resposta Hidrológica (HRU’s), que são 
partes da sub-bacia que possuem uma única combinação de uso do solo/solo/manejo, fornecendo uma descrição física melhor do balanço hídrico (Arnold et al., 1998).

O SWAT, entretanto, apresenta algumas limitações, como o fato de utilizar a Equação Modificada de Perda de Solo-MEUPS, para o cálculo da produção de sedimentos na bacia, mesmo sabendo que essa é uma equação de perda de solo, que se processa na sua maior parte como erosão laminar (Machado, 2002). Além do mais, o software não interpreta chuvas intensas em curtos períodos (Moro, 2005) e não realiza um balanceamento completo de massa dos componentes que simulam o processo de água subterrânea (Machado, 2002). Mas a grande limitação do SWAT é a dificuldade de se encontrar parâmetros regionais que alimentem o seu banco de dados.

No Brasil alguns trabalhos foram realizados aplicando-se o SWAT como ferramenta para os estudos hidrológicos e de produção de sedimentos, tais quais os de Oliveira (1999), Machado (2002), Barsanti et al. (2003), Moro (2005), Prado (2005), Neves (2005), Baldissera (2005), Minoti (2006), Adriolo et al. (2008), Blainski et al. (2008), Baltokoski (2008), Lopes (2008) e Lino (2009). Entretanto, não há um grupo de estudos formado no país que dê continuidade à modelagem ambiental em áreas agrícolas, áreas cujas atividades promovem grande impacto no meio ambiente.

Neste trabalho é simulada a erosão produzida na bacia hidrográfica do ribeirão São Bartolomeu, uma bacia hidrográfica tipicamente agrícola, considerando diferentes cenários de ocupação e uso do solo, a partir da utilização do modelo SWAT. A modelagem e simulação de cenários nessa área, localizada na Zona da Mata de Minas Gerais, é particularmente importante por não haver nenhum tipo desse estudo na região, que é representativa das áreas de planalto dissecado do mar de morros que se repetem no território brasileiro, em sua fachada Atlântica.

\section{MATERIAL E MÉTODOS}

A quantificação das perdas de água e solo foi realizada a partir de parcelas experimentais submetidas a chuvas naturais, implantadas em uma região da bacia hidrográfica do ribeirão São Bartolomeu (BHRSB), na Zona da Mata de Minas Gerais, região Sudeste do Brasil. Os dados de solo, vegetação e clima foram obtidos a partir de ensaios com amostras coletadas "in situ”, estudos e informações da região.

\subsection{Escolha da área de estudo}

A bacia hidrográfica do ribeirão São Bartolomeu é uma sub-bacia do rio Piranga, a qual é uma sub-bacia do rio Doce, como mostrado na Figura 1, e ocupa uma área de 5.422 ha, representando cerca de $18 \%$ da área do município de Viçosa, MG.

A bacia do ribeirão São Bartolomeu faz parte de uma região crítica do estado de Minas Gerais, apresentando baixa produtividade animal, agricultura pouco dinâmica, solos frágeis de baixa fertilidade e bastante degradados (Cardoso, 2003; Souza, 2004; Santos, 2007). Bacias como essa, tipicamente agrícola, carecem de estudos de modelagem ambiental para subsidiar ações voltadas à realização do zoneamento agroecológico, agregando aspectos ambientais e socioeconômicos da região. 


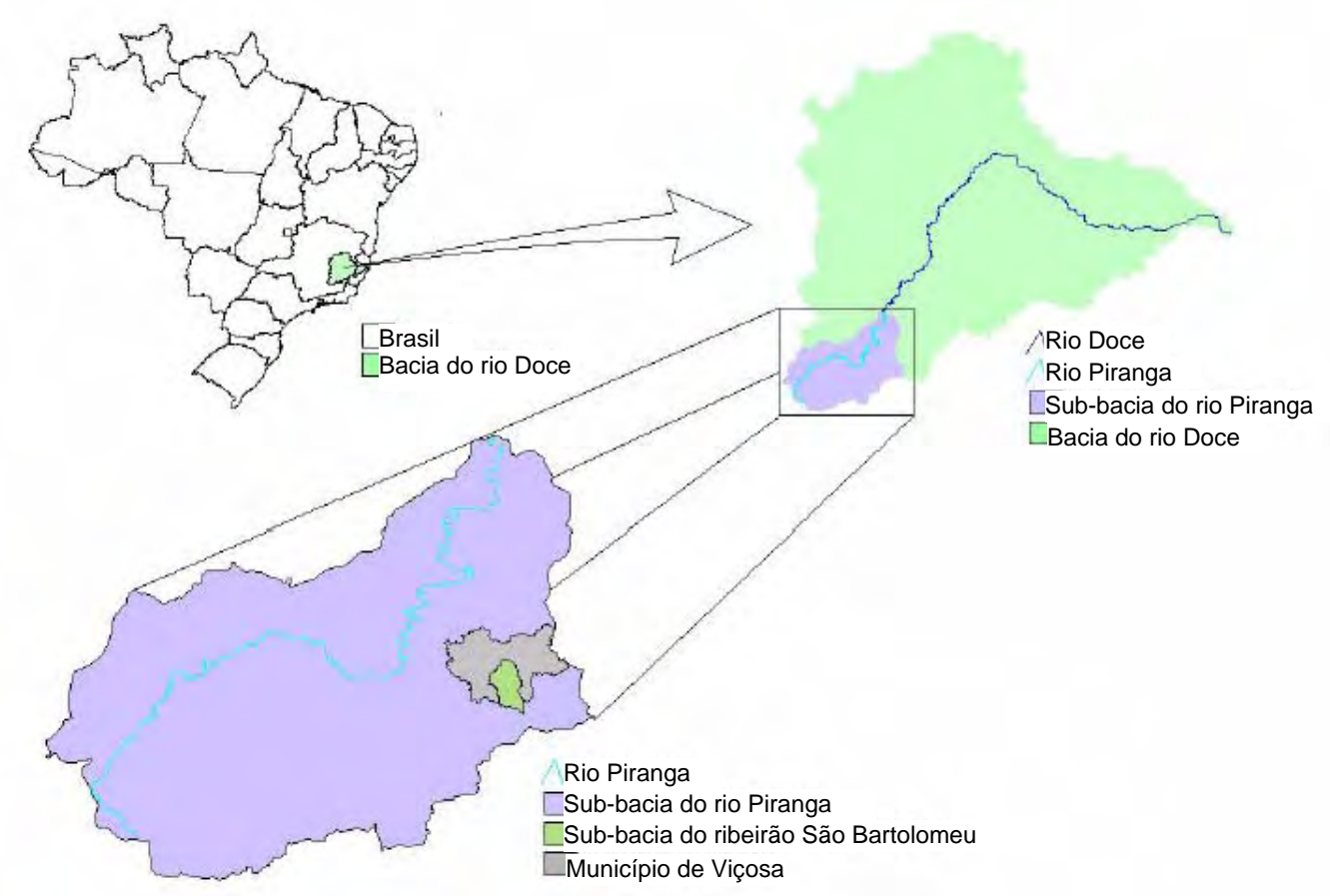

Figura 1. Localização da sub-bacia do ribeirão São Bartolomeu no contexto da bacia do rio Doce e da sub-bacia do rio Piranga.

\subsection{Caracterização da bacia}

A bacia do ribeirão São Bartolomeu, de área igual a 54,2 km², tem sua maior parte ocupada por pastagens, somando mais de 38\% da área e distribuídas por toda a região, conforme mostra a Tabela 1. Esses dados foram obtidos da carta de uso e ocupação do solo, apresentada na Figura 2, confeccionada a partir da digitalização em tela no AutoCad da Imagem IKONOS de 2008 da bacia e validada por meio de visitas a campo por toda a área.

Tabela 1. Distribuição da área total em relação ao uso do solo da bacia hidrográfica do ribeirão São Bartolomeu.

\begin{tabular}{l|cc}
\hline Uso do Solo & Área $\left.\mathbf{( k m}^{\mathbf{2}}\right)$ & $\mathbf{( \% )}$ \\
\hline Solo exposto & 0,38 & 0,7 \\
Pastagem não degradada & 12,90 & 23,8 \\
Pastagem degradada & 8,02 & 14,8 \\
Eucalipto & 0,54 & 1,0 \\
Mata secundária alta & 14,80 & 27,3 \\
Mata secundária baixa & 2,60 & 4,8 \\
Café & 3,14 & 5,8 \\
Área urbana & 10,08 & 18,6 \\
Agricultura em geral & 1,36 & 2,5 \\
Hidrografia & 0,38 & 0,7 \\
\hline Área Total & $\mathbf{5 4 , 2} \mathbf{~ k m}^{\mathbf{2}}$ & $\mathbf{1 0 0 , 0}$ \\
\hline
\end{tabular}




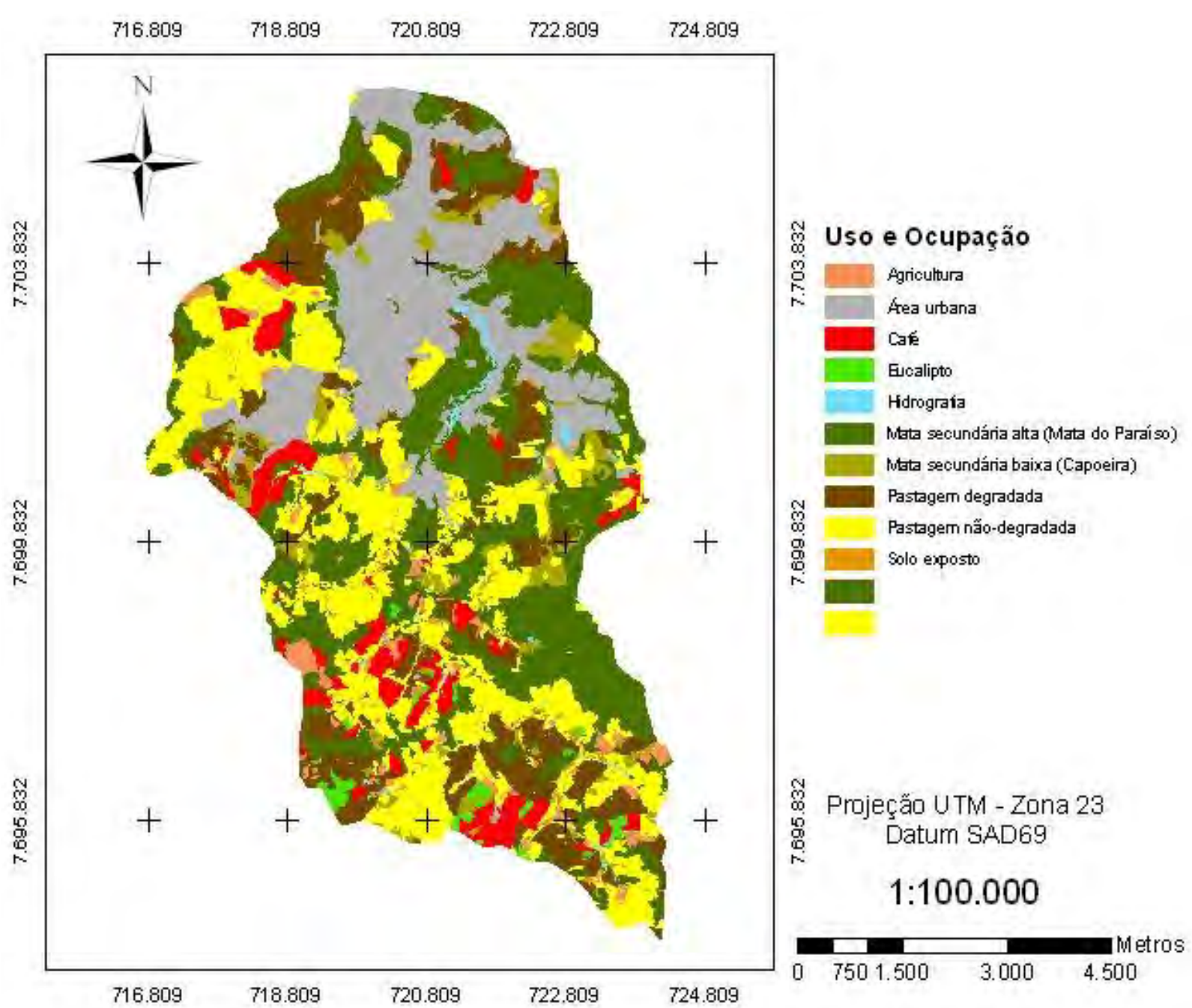

Figura 2. Carta de uso do solo da bacia hidrográfica do ribeirão São Bartolomeu.

Quanto à carta de solos, ela foi obtida a partir da análise e descrição de perfis de solos, interpretação de fotografias aéreas, imagens IKONOS e do bloco-diagrama da paisagem da bacia, encontrado em Schaefer et al. (1998). Foram identificadas 4 classes principais na bacia, baseadas em EMBRAPA (2006), conforme apresentadas na Tabela 2 e na Figura.3.

Tabela 2. Classes de Solo existentes na bacia hidrográfica do ribeirão São Bartolomeu.

\begin{tabular}{|c|c|c|}
\hline Classe de Solo & Área $\left(\mathrm{km}^{2}\right)$ & $(\%)$ \\
\hline Cx- Cambissolo & 8,13 & 15,0 \\
\hline PVA (PVAd1, PVAd2, PVAd3)- Argissolo Vermelho Amarelo & 18,21 & 33,6 \\
\hline Gx - Gleissolo & 2,76 & 5,1 \\
\hline LVA (LVAd1, LVAd2)- Latossolo Vermelho Amarelo & 25,09 & 46,3 \\
\hline Área Total & $54,2 \mathbf{k m}^{2}$ & 100,0 \\
\hline
\end{tabular}




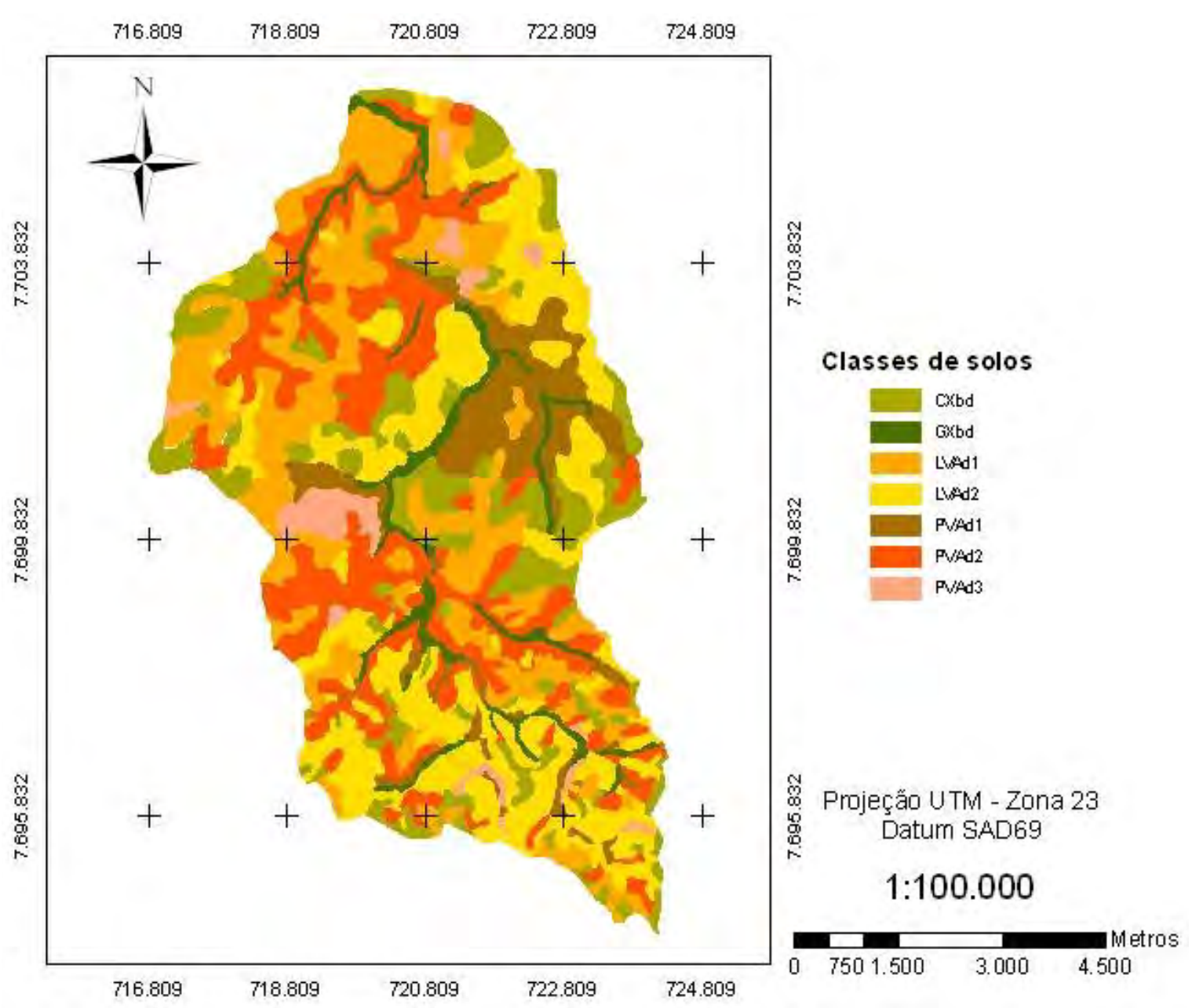

Figura 3. Carta de solos da bacia hidrográfica do ribeirão São Bartolomeu.

Quanto às características físicas, várias delas foram retiradas do Modelo Digital de Elevação Hidrologicamente Consistente - MDEHC (Figura 4). O MDEHC foi gerado a partir do interpolador TOPOGRID do ARC/Info, utilizando-se a hidrografia da bacia (mapas em escala 1:50.000), simplificada e orientada na direção do escoamento, e da altimetria (mapas em escala 1:50.000), com curvas de nível de 20 em $20 \mathrm{~m}$, eliminando-se as depressões espúrias, aprofundando o relevo ao longo da hidrografia e garantindo a consistência do escoamento superficial ao longo da rede de drenagem gerada.

A Tabela 3 mostra algumas dessas características físicas retiradas a partir do MDEHC da bacia do ribeirão São Bartolomeu.

Tabela 3. Características físicas da bacia do Ribeirão São Bartolomeu.

\begin{tabular}{ll}
\hline Área $(\mathrm{A})$ & $54,22 \mathrm{~km}^{2}$ \\
Perímetro (P) & $36,10 \mathrm{~km}$ \\
Comprimento axial (L) & $20.114,13 \mathrm{~m}$ \\
Índice de Compacidade ( $\left.\mathrm{K}_{\mathrm{c}}\right)$ & 1,37 \\
Î́dice de Forma $\left(\mathrm{K}_{\mathrm{f}}\right)$ & 0,13 \\
Índice de Circularidade (IC) & 0,52 \\
Densidade de drenagem (Dd) & 3,11 \\
Altitude média & $730 \mathrm{~m}$ \\
Declividade média & $12,8 \%$ \\
Comprimento do curso d'água principal & $19483,50 \mathrm{~m}$ \\
Declividade do curso d'água principal & $0,000116 \mathrm{~m} / \mathrm{m}$ \\
\hline
\end{tabular}




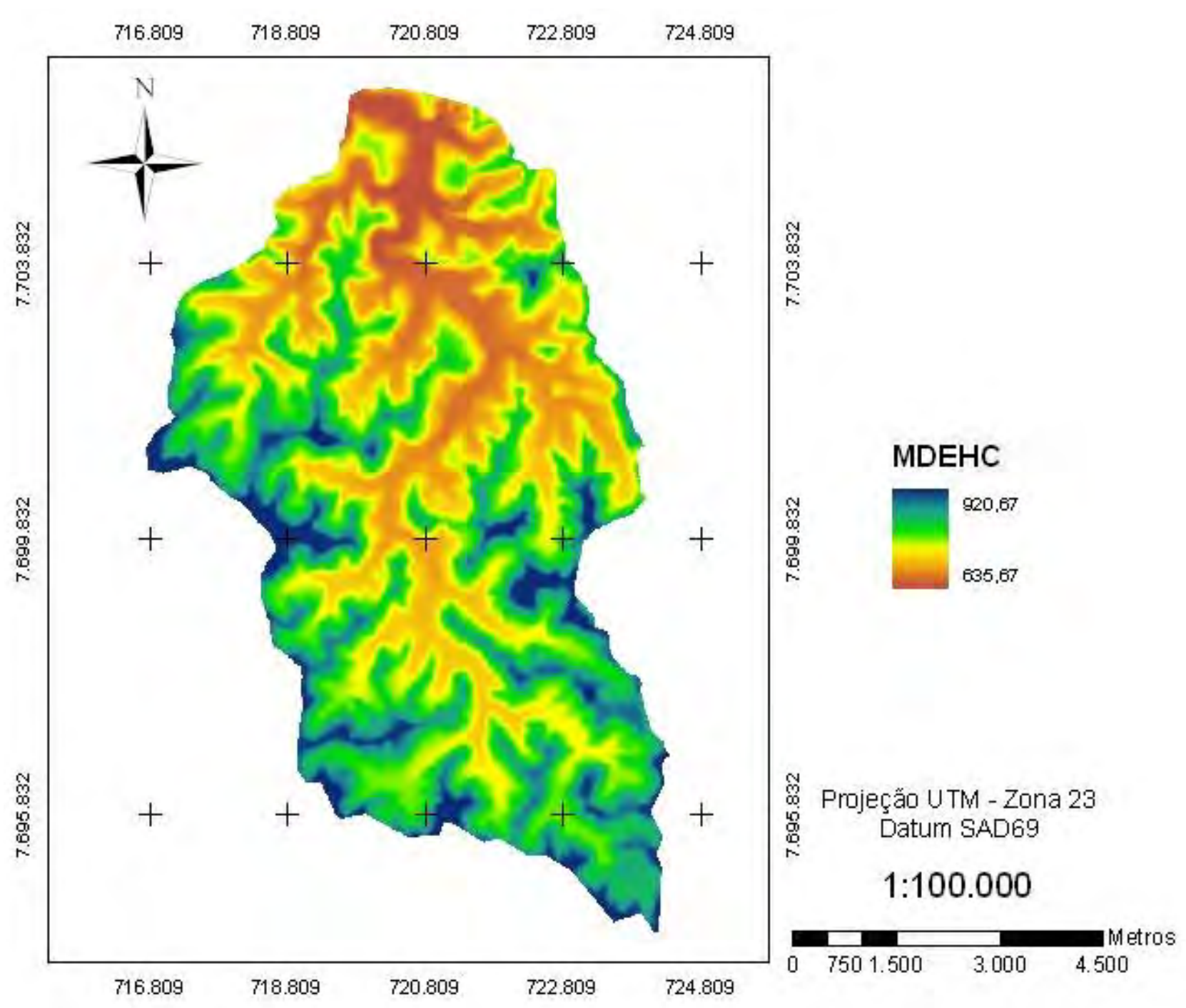

Figura 4. Modelo Digital de Elevação Hidrologicamente Consistente da BHRSB.

\subsection{Parcelas experimentais}

O monitoramento do escoamento superficial e das perdas de solo, sob condições de chuva natural, foi realizado entre dezembro/2006 a março/2008, em 10 parcelas experimentais com área de captação de chuva de $40 \mathrm{~m}^{2}$ e estrutura coletora de sedimentos e de medição de escoamento superficial, conforme Figura 5. A escolha dos locais onde as parcelas foram instaladas teve como base a declividade das encostas e o tipo de cobertura vegetal, de forma a abranger as principais características das culturas existentes na bacia.

Para cada evento pluviométrico ocorrido foi calculado o escoamento superficial a partir dos linígrafos instalados nos vertedouros das parcelas, que forneciam informações para a determinação da lâmina d'água escoada. Já as perdas de solo provocadas pela força do escoamento foram quantificadas pelo método direto (Bertoni e Lombardi Neto, 1990). A lâmina de escoamento superficial, calculada pela relação entre o volume total escoado, obtido pelos linígrafos, a área da parcela experimental e o solo retido nas estruturas de coleta, em t/ha, serviram de base para a calibração do modelo. 


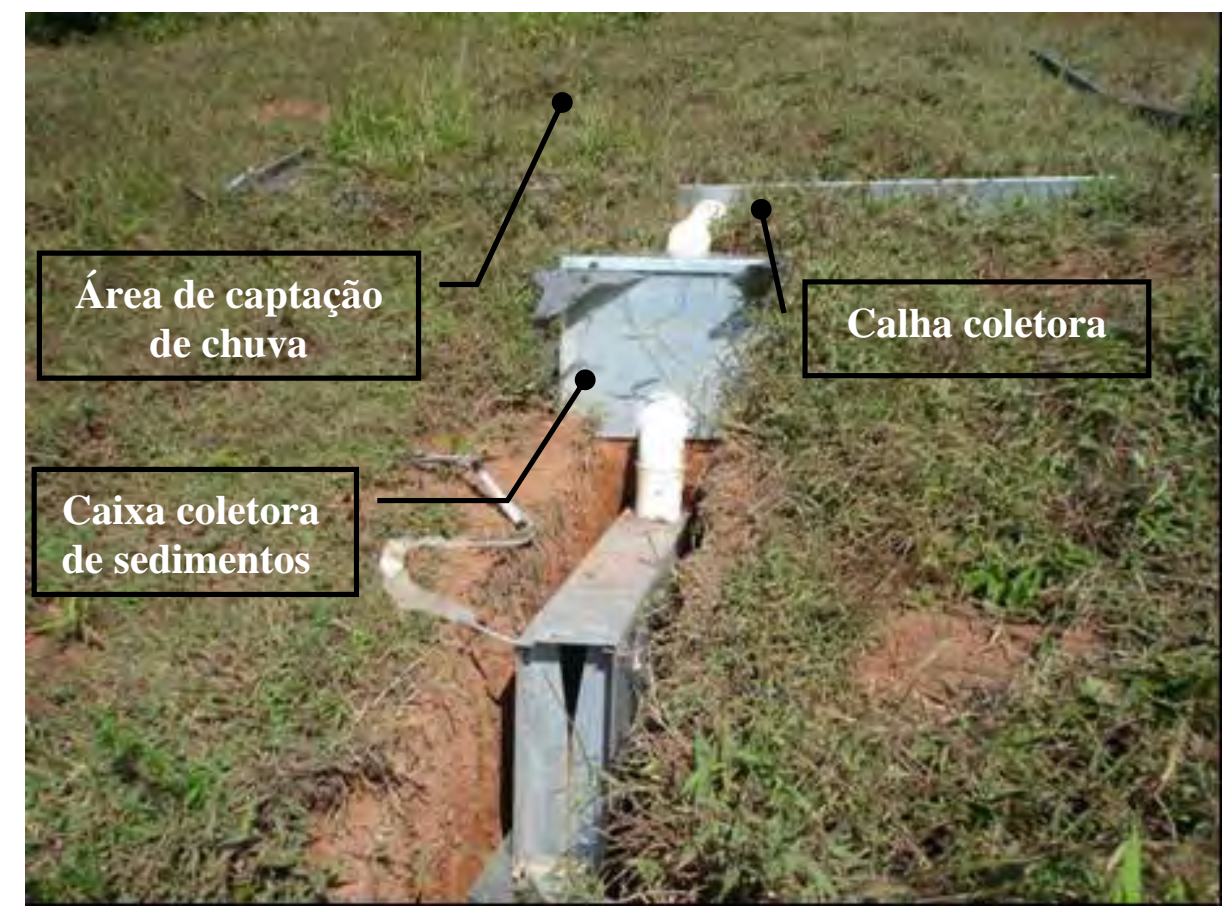

Figura 5. Parcelas experimentais compostas de área de captação da chuva, calha coletora e tubo de PVC para condução do escoamento superficial para a estrutura de coleta das perdas de solo e água, composta por um sistema de filtragem, no qual os sedimentos transportados pelo escoamento superficial eram retidos no filtro e o escoamento superficial era conduzido para uma caixa retangular. Essa caixa apresentava, na sua extremidade final, um vertedouro triangular utilizado para a medição da vazão de escoamento superficial.

\subsection{Dados de entrada no SWAT}

Dentre as informações espaciais que devem ser fornecidas ao SWAT estão o Modelo Digital de Elevação Hidrologicamente Consistente - MDEHC, a Carta de Solos e a Carta de Uso e Ocupação. Além deles, existe uma série de dados tabulares relacionados ao solo, clima, fisiologia de culturas e tipos de ocupações agrícolas e práticas de manejo do solo que devem ser fornecidos ao modelo, os quais foram obtidos a partir de dados históricos da região, ensaios laboratoriais, estudos e referências literárias.

Para a estimativa do escoamento superficial, adotou-se o método do número da curva (CN) do SCS (Soil Conservation Service). Os valores de CN foram obtidos para cada combinação de classe e cobertura existente, segundo as HRU's formadas, em condições de umidade antecedente AMC II.

A evapotranspiração foi calculada pelo método de Penman-Monteith, que segundo Smith (1991), é o que melhor descreve o fenômeno.

\section{RESULTADOS E DISCUSSÃO}

A bacia do ribeirão São Bartolomeu é representativa de bacia rural, com ocupação urbana, que vem, ao longo dos anos, passando por processos erosivos. Áreas que deveriam estar reflorestadas nas encostas estão inadequadamente ocupadas, pastagens e plantações agrícolas encontram-se em áreas de preservação permanente e não existe isolamento dessa faixa de preservação. As declividades elevadas, agregadas à falta de práticas conservacionistas, favorecem a ocorrência de enxurrada, erosão e lavagem de nutrientes, empobrecimento do solo, assoreamento dos cursos d'água, potencializando a ocorrência de 
enchentes, associados a uma série de problemas ambientais urbanos, conforme também discorreram Vilela (1998), Vilela e Romanovzki (2001) e Souza (2004).

A instalação das parcelas experimentais permitiu desenvolver um programa de monitoramento para a identificação e quantificação de fontes de produção de sedimentos na bacia. A partir das parcelas experimentais foi possível verificar o comportamento dos solos e culturas da região quanto à produção de sedimentos e escoamento superficial, em dois períodos chuvosos, e com isso subsidiar a análise dos resultados da modelagem ambiental.

Em todas as parcelas foi evidenciada a influência da intensidade da precipitação, associada à umidade do solo, na produção de sedimentos, isto é, as perdas quantificadas em todas as parcelas foram função, também, da ocorrência de chuva no dia anterior. Isso quer dizer que precipitações equivalentes produziram quantidades de perda de solo distintas, dependentes das chuvas ocorridas nos dias anteriores, que alteraram as condições da superfície, como já haviam destacado em seus trabalhos Bertoni e Lombardi Neto (1990), Mermut et al. (1997), Amorim (2004), Brandão et al. (2007), Carvalho (2008), Lopes (2008), dentre outros autores. Os resultados médios para a bacia, relacionados tanto com a lâmina precipitada quanto com a intensidade de precipitação e, levando-se em conta a proporção ocupada na bacia pela cultura da parcela experimental, estão apresentados nas Figuras 6 e 7, respectivamente.

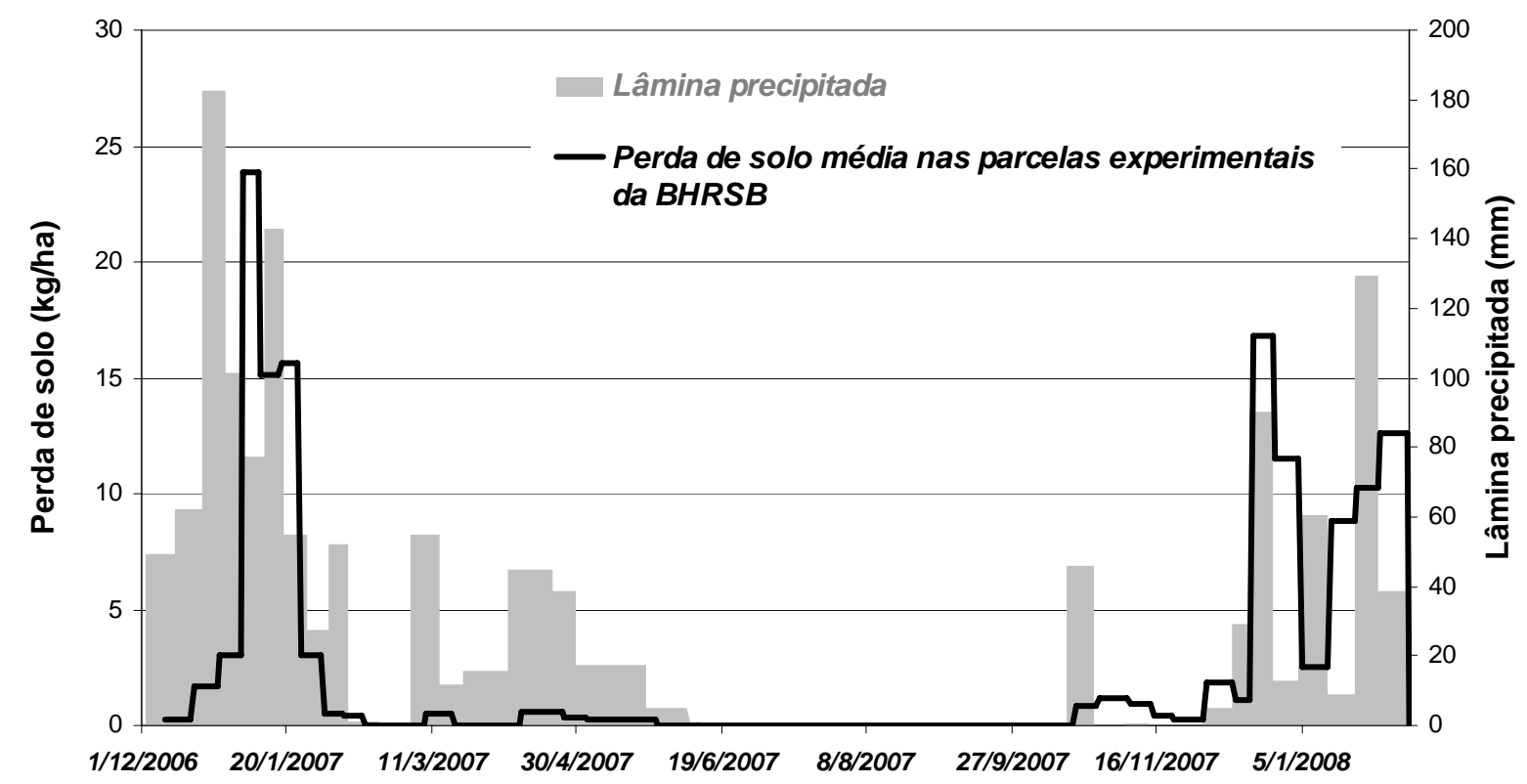

Figura 6. Comparação entre a lâmina precipitada e perda de solo média nas parcelas experimentais da bacia hidrográfica do ribeirão São Bartolomeu. 


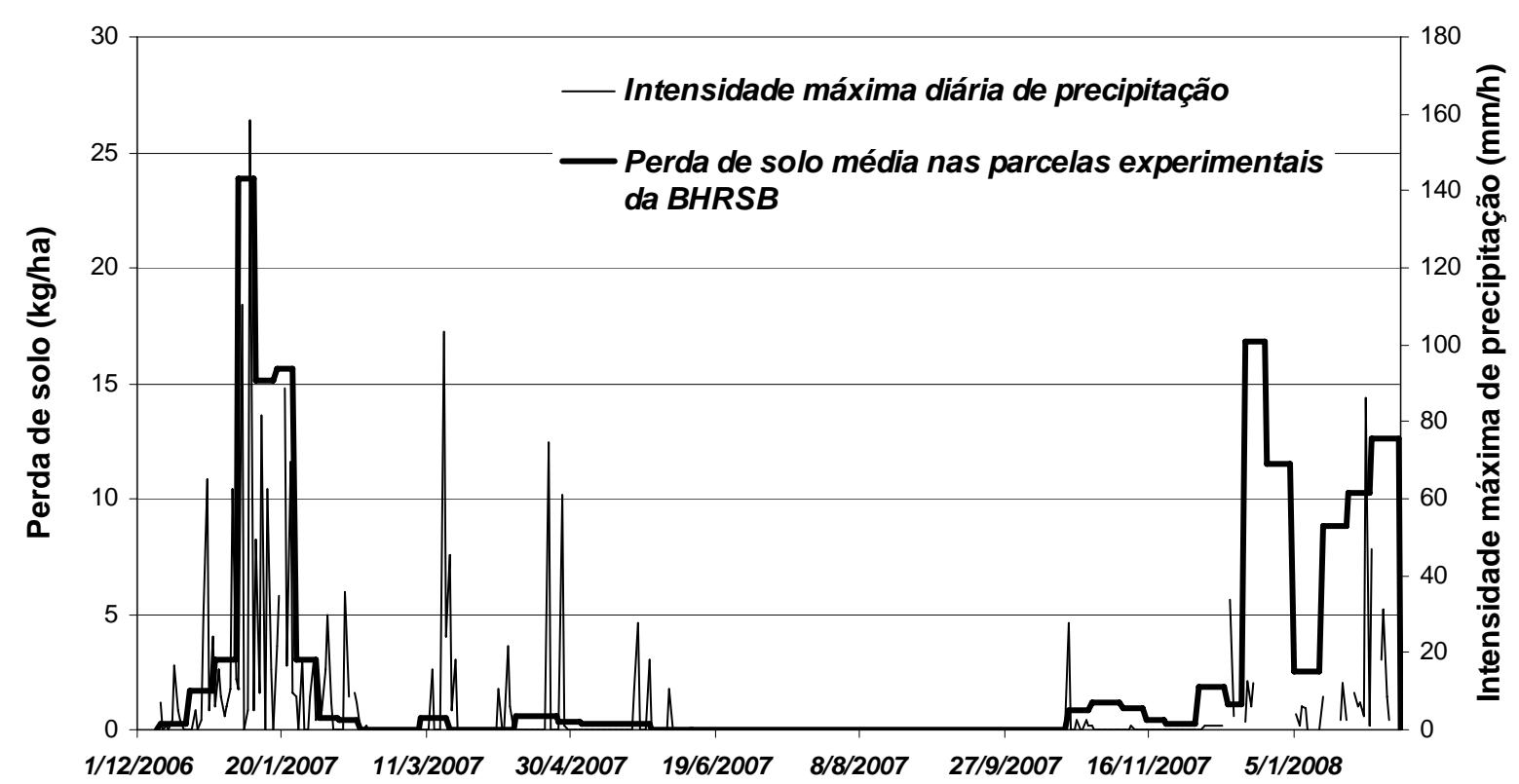

Figura 7. Comparação entre intensidade de precipitação e perda de solo média nas parcelas experimentais da bacia hidrográfica do ribeirão São Bartolomeu.

No SWAT, a simulação da produção de sedimentos e escoamento superficial na área foi realizada dividindo-se a bacia em 73 sub-bacias com, no máximo, 40 hectares cada. Esse tamanho foi considerado adequado, de acordo com a resposta obtida pelo modelo na calibração, cujo desempenho foi avaliado pelo coeficiente de eficiência (COE) de Nash e Sutcliffe. (Figura 8).

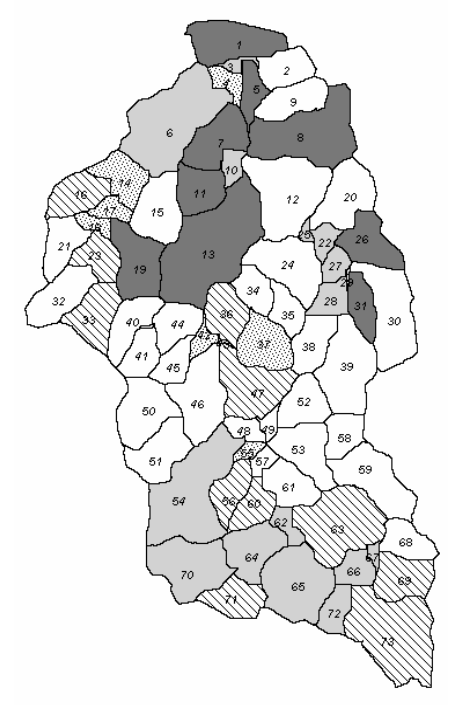

2006

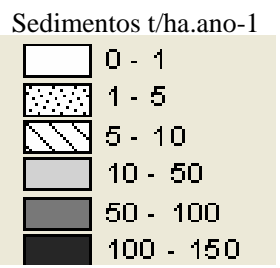

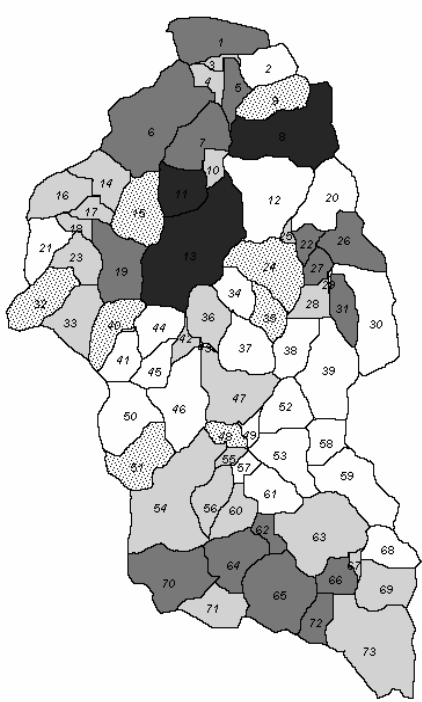

2007

Sedimentos t/ha.ano-1

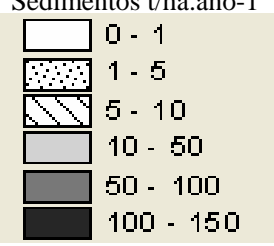

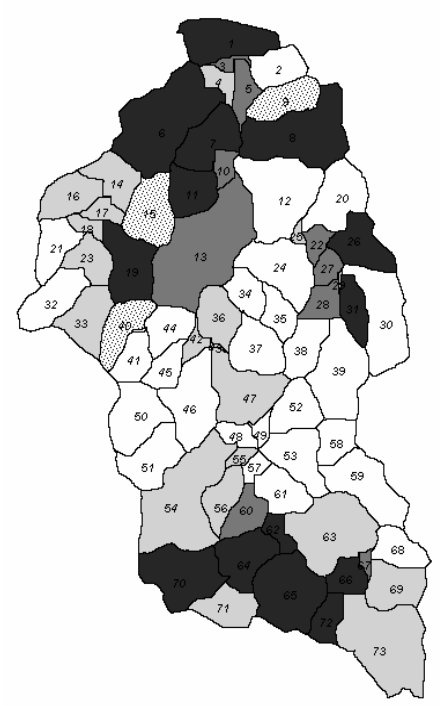

2008

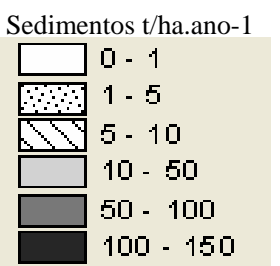

Figura 8. Simulação da produção de sedimentos, em t/ha.ano-1, na BHRSB para os anos de 2006, 2007 e 2008, no cenário original de uso e ocupação do solo. 
A partir do modelo calibrado, foi possível estimar, dentre outros fatores, a produção de sedimentos, sólidos em suspensão, precipitação, percolação, escoamento superficial e evapotranspiração para a bacia. Neste trabalho, atenção maior foi dada à produção de sedimentos, a qual está apresentada, ano a ano, em cada sub-bacia e para as condições reais de ocupação do solo.

Verificou-se na modelagem que a área ao norte, onde predomina a parte urbana, é a maior produtora de sedimentos na bacia, alcançando 82, 109 e $138 \mathrm{t} / \mathrm{ha}^{-a n o^{-1}}$, nos anos de 2006, 2007 e 2008, respectivamente. Esse resultado é mais que esperado, haja vista o grande volume de material que obstrui e entope as vias pluviais nos períodos de chuva que atingem a cidade de Viçosa. As menores perdas de solo em todos os 3 anos de simulação ocorreram na parte intermediária da bacia, onde predomina uma mata secundária. Esse tipo de cobertura promove maior interceptação das gotas de chuva pelo dossel da mata, conferindo maior proteção ao solo, além da existência de serapilheira, gerando maiores teores de matéria orgânica no solo, agregados de maior estabilidade, melhor estruturação e maior permeabilidade.

A produção de sedimentos tende a elevar-se em direção à nascente, numa região ao sul da bacia, onde predominam pastagens. Esse tipo de ocupação tende a aumentar progressivamente na região, conforme análises espaciais comparativas, realizadas com Imagens Ikonos de 2003 e 2008, as quais demonstraram que em 5 anos houve um acréscimo de aproximadamente $2 \%$ na área ocupada por pastagens. Essa progressiva ocupação é de grande preocupação e levanta a discussão para a necessidade de recuperação da cabeceira e atenção para outras regiões da bacia, que podem passar a contribuir de forma bem mais acentudada na produção de sedimentos. Sendo assim, uma antecipação das medidas corretivas a serem tomadas pode ser obtida a partir da análise de um novo cenário, substituindo parte das áreas de pastagens por plantações de eucalipto, por exemplo.

Assim, além de se estudar a situação atual da área, cenários com base em possíveis mudanças em relação ao uso e ocupação do solo foram também simulados, como aquele citado, substituindo a área de pastagens degradadas por eucalipto (Figura 9). Pelas simulações realizadas, observou-se significativa redução na produção de sedimentos em toda bacia, em especial na sua cabeceira, onde foi realizada a maior parte das substituições. Nas sub-bacias dessa região, no cenário original, a perda de solo média para os três anos de simulação foi de $68 \mathrm{t} / \mathrm{ha} \mathrm{ano}^{-1}$, enquanto que para o cenário simulado esse valor decaiu para cerca de 20 t/ha.ano ${ }^{-1}$, sugerindo que há eficiência nesse tipo de manejo e cobertura do solo no controle do processo erosivo. Para toda a bacia, representando uma substituição de $14,8 \%$ de pastagens em eucalipto, embora a queda não tenha sido tão acentuada, a modelagem indicou que houve uma diminuição da média produzida de sedimentos nos três anos de 33 t/ha.ano ${ }^{-1}$ para 26 t/ha.ano ${ }^{-1}$, entre o cenário original e o simulado. 


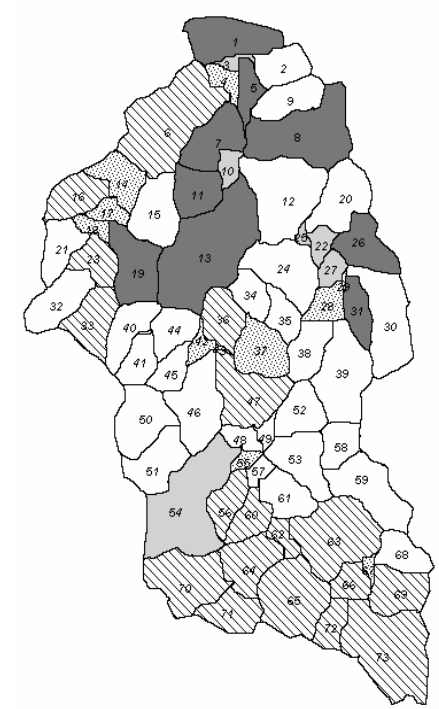

2006

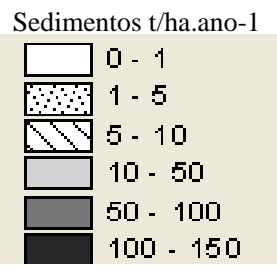

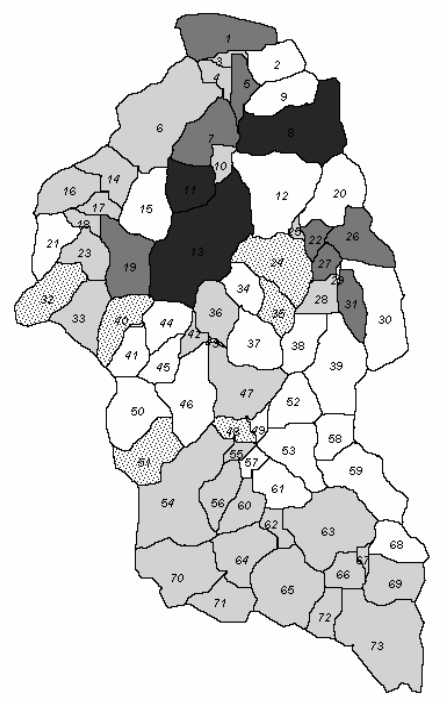

2007

Sedimentos t/ha.ano-1

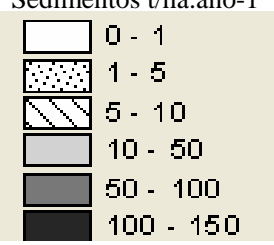

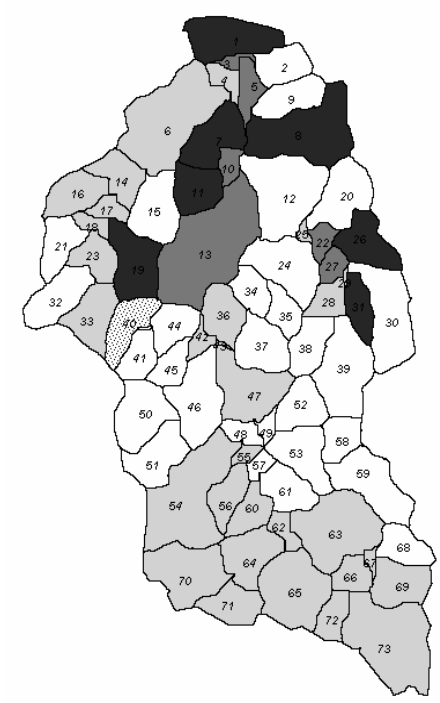

2008

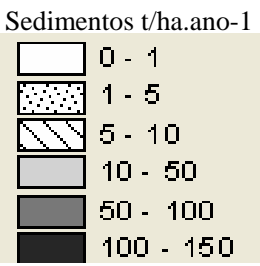

Figura 9. Simulação de cenário para produção de sedimentos, em t/ha.ano-1, na BHRSB em 2006, 2007 e 2008, no cenário simulado de substituição das pastagens degradadas por eucalipto.

Complementando a análise das condições que favorecem a perda de solo na área, nas parcelas experimentais também foi monitorado o escoamento superficial, diariamente, por meio dos linígrafos, o que tornou possível conhecer o volume total escoado em cada parcela a cada evento pluviométrico e verificar o balanço hídrico das áreas. A partir dos dados obtidos em campo e da simulação pelo SWAT, determinou-se o coeficiente de escoamento superficial para cada parcela e das ocupações predominantes. Na Tabela 4, apresentam-se os valores calculados e estimados durante o período em que as parcelas permaneceram em campo.

Tabela 4. Coeficientes de escoamento superficial determinados a partir de dados de campo e simulados pelo SWAT.

\begin{tabular}{|c|c|c|c|c|c|c|}
\hline \multirow{3}{*}{$\begin{array}{l}\text { Uso e } \\
\text { Ocupação }\end{array}$} & \multicolumn{6}{|c|}{ Coeficiente de Escoamento Superficial } \\
\hline & \multicolumn{2}{|c|}{2006} & \multicolumn{2}{|c|}{2007} & \multicolumn{2}{|c|}{2008} \\
\hline & $\begin{array}{c}\text { CAMPO } \\
\text { (a partir de } \\
01 / 12 / 06 \text { ) }\end{array}$ & SWAT & CAMPO & SWAT & $\begin{array}{c}\text { CAMPO } \\
\text { (até } \\
\text { 01/03/2008) }\end{array}$ & SWAT \\
\hline Café A & 0,02 & & 0,02 & & 0,04 & \\
\hline Café B & 0,06 & 008 & 0,03 & 007 & 0,05 & \\
\hline Café Convexo & 0,03 & 0,08 & 0,04 & $0,0 /$ & 0,03 & 0,24 \\
\hline Café Côncavo & 0,05 & & 0,29 & & 0,10 & \\
\hline Pasto Convexo & 0,03 & & 0,06 & & 0,04 & \\
\hline Pasto Côncavo & 0,25 & & 0,18 & & 0,20 & \\
\hline Pasto Convexo Degradado* & - & 0,07 & 0,02 & 0,05 & 0,01 & 0,18 \\
\hline Pasto Côncavo Degradado* & - & & 0,01 & & 0,01 & \\
\hline Mata & 0,05 & 0,04 & 0,04 & 0,02 & 0,05 & 0,10 \\
\hline Eucalipto & 0,01 & & 0,02 & & 0,02 & \\
\hline Área Urbana & - & 0,66 & - & 0,69 & - & 0,83 \\
\hline
\end{tabular}

*Início das coletas em out/2007. 
Observa-se que os valores determinados em campo e simulados pelo SWAT são bem próximos e compatíveis com as realidades das culturas comparadas, exceto para a área urbana, da qual não se têm dados de campo e não se pôde fazer tal comparação, sendo o modelo considerado satisfatoriamente aplicado. Além do mais, os valores ratificam os resultados obtidos com a modelagem, ao serem determinados, em geral, maiores valores de coeficiente de escoamento superficial para as pastagens, o que proporciona maior perda de solo.

Entretanto, mesmo com uma boa aproximação dos resultados, é importante ficar atento a alguns aspectos, como, por exemplo, na escolha das CN's, as quais podem gerar erros significativos, mesmo com pequenas variações. Nesse sentido, a aplicação de outro método para avaliar o escoamento superficial, como o Método de Green e Ampt, pode ser particularmente interessante para analisar melhor os resultados e dar credibilidade aos valores determinados na modelagem.

Outra constatação importante a respeito das limitações do modelo SWAT é o fato de que ele não considera diferentes estágios e condições das diversas culturas na bacia. Dessa forma, pastagens degradadas foram consideradas idênticas quanto ao nível de degradação, bem como a cobertura de eucalipto, que apresenta diversos estágios de desenvolvimento na área da pesquisa.

\section{CONCLUSÃO}

Nas condições edafoclimáticas da bacia do ribeirão São Bartolomeu, no município de Viçosa, MG, e nas demais condições de realização do experimento, pode-se dizer que:

O SWAT foi eficaz, não só na caracterização da produção de sedimentos na área de estudo, identificando áreas mais vulneráveis e críticas, mas também na simulação de cenários ambientais, dentre eles aqueles menos impactantes ao ambiente, que combinam melhores técnicas de manejo e cobertura do solo.

As áreas ocupadas por pastagens foram consideradas as de maior impacto ao ambiente quando se analisa a produção de sedimentos.

As perdas de solo são dependentes da frequência das precipitações, que alteraram as condições da superfície favorecendo ou não a ocorrência do escoamento superficial e consequente perda de solo.

Pela análise dos dados das parcelas experimentais e com o SWAT foi possível calibrar o modelo e avaliar os impactos potenciais decorrentes da ocupação atual da bacia e da alteração de suas características hidrossedimentológicas, a partir da simulação de substituição de parte da cobertura de pastagens por eucalipto. Nessa simulação, houve redução da perda de solo na região da cabeceira em quase 4 vezes, refletindo numa redução em $21 \%$ na produção de sedimentos de toda a bacia.

Mesmo sabendo que o emprego de um modelo conceitual requer experiência, maturidade e acúmulo de conhecimento para a obtenção de resultados coerentes e confiáveis, a começar pelos dados utilizados, como os CN's, o primeiro trabalho com esse enfoque nessa área apresentou resultados satisfatórios e validados para condições de campo, a partir dos dados obtidos nas parcelas experimentais instaladas nas culturas predominantes da bacia.

\section{REFERÊNCIAS}

ADRIOLO, M. V.; SANTOS, I.; GIBERTONI, R. C.; CAMARGO, A. S. G. Calibração do modelo SWAT para a produção e transporte de sedimentos. In: SIMPÓSIO BRASILEIRO SOBRE PEQUENAS E MÉDIAS CENTRAIS HIDRELÉTRICAS, 6., 2008, Belo Horizonte. Anais... Rio de Janeiro: CBDB, 2008. 
AMORE, E.; MODICA, C.; NEARING, M. A.; SANTORO, V. C. Scale effect in USLE and WEPP application for soil erosion computation from three Sicilian basins. Journal of Hydrology, v. 293, p. 100-114, 2004.

AMORIM, R. S. S. Desprendimento e arraste de partículas de solo decorrentes de chuvas simuladas. 2000. 74f. Dissertação (Mestrado em Engenharia Agrícola) - Universidade Federal de Viçosa, Viçosa, MG, 2000.

AMORIM, R. S. S. Avaliação dos modelos de predição da erosão hídrica USLE, RUSLE e WEPP para condições edafoclimáticas brasileiras. 2004. 120f. Dissertação (Doutorado em Engenharia Agrícola) - Universidade Federal de Viçosa, Viçosa, MG, 2004.

ARABI, M.; FRANKENBERGER, J. R.; ENGEL, B. A.; ARNOLD, J. G. Representation of agricultural conservation practices with SWAT. Hydrol. Process., v. 22, n. 16, p. 304255, 2008.

ARNOLD, J. G.; WILLIAMS, J. R.; NICKS, A. D.; SAMMONS, N. B. SWRRB: a basin scale simulation model for soil and water resources management. College Station: Texas A\&M Univ. Press, 1990. 115p.

ARNOLD, J. G.; WILLIAMS, F. R.; MAIDMENT, D. R. Continuous-time water and sediment routing model for large basins. Hydr. Engin., v. 121, p.171-183, 1995.

ARNOLD, J. G.; SRINIVASAN, R.; MUTTIAH, R. S.; WILLIAMS, J. R. Large area hydrologic modeling and assessment. Part I: model development. JAWRA, v. 34, n. 1, p. 73-89, 1998.

BALDISSERA, G. C. Aplicabilidade do modelo de simulação hidrológica SWAT (Soil and Water Assessment Tool) para a bacia hidrográfica do Rio Cuiabá, MT. 2005. 132f. Dissertação (Mestrado em Física do Meio Ambiente) - Universidade Federal do Mato Grosso, Cuiabá, 2005.

BALTOKOSKI, V. Modelo SWAT2005 aplicado às sub-bacias dos rios Conrado e Pinheiro - Pato Branco/PR. 2008. 120f. Dissertação (Mestrado em Engenharia Agrícola) - Universidade Estadual do Oeste do Paraná, Cascavel, 2008.

BARSANTI, P.; DISPERATI, L.; MARRI, P.; MIONE, A. Soil erosion evaluation and multitemporal analysis in two Brazilian basins. In: INTERNATIONAL SWAT CONFERENCE, 2., 2003, Bari. Reports... College Station: TWRI, 2003.

BLAINSKI, E.; SILVEIRA, F. A.; CONCEIÇÃO, G. Utilização do modelo hidrológico SWAT para estudos na microbacia hidrográfica do rio Araranguá/SC. In: TALLER INTERNATIONAL RED RIEGOS CYTED, 2008, Florianópolis. Anais... Florianópolis: CEER, 2008.

BERTONI, J.; LOMBARDI NETO, R. Conservação do solo. São Paulo: Ícone, 1990. 355p.

BIESBROUCK, B.; WYSEURE, G.; VAN ORSCHOVEN, J.; FEYEN, J. AVSWAT 2000. Leuven: K.U.Leuven, 2002. 199p.

BRANDÃO, V. S.; SILVA, D. D.; RUIZ, H. A.; PRUSKI, F. F.; SCHAEFER, C. E. G. R.; MARTINEZ, M. A. et al. Perdas de solo e caracterização física e micromorfológica de crostas formadas em solos sob chuva simulada. Eng. Agríc., Jaboticabal, v. 27, n. 1, p. 129-138, jan./abr. 2007. 
CARDOSO, I. M. Sistemas agroflorestais na zona da mata de Minas Gerais: um processo contínuo de aprendizado. In: SEMINÁRIO SISTEMAS AGROFLORESTAIS E DESENVOLVIMENTO SUSTENTÁVEL, 2003, Campo Grande. Textos Integrais... Disponível em: <http://saf.cnpgc.embrapa.br/07publicacoes.html>. Acesso: maio 2010.

CARVALHO, N. O. Hidrossedimentologia prática. 2. ed. Rio de Janeiro: Interciência, 2008. 600p.

CHRISTOFOLETTI, A. Modelagem de sistemas ambientais. São Paulo: Edgard Blutcher, 1999. $236 \mathrm{p}$.

DIEZ R., T. Diseño e implementacion de una aplicacion como soporte para toma de decisiones en el estudio de cuencas hidrograficas (Data Basin). In: Memoria CONDESAN 2002. Quinta Parte: análisis de políticas en los Andes Disponível em: $<$ www.infoandina.org/system/files/recursos/REG0162002.pdf>. Acesso: maio 2010.

DUARTE, P.; GUERREIRO, M. J.; REIA, J.; FONSECA, L. C.; PEREIRA, A.; AZEVEDO, B. et al. Gestão de zonas costeiras: aplicação à Ria Formosa (Sul de Portugal). Revista Ciência Agronômica, v. 38, n. 1, p. 118-128, 2007.

EMPRESA BRASILEIRA DE PESQUISA AGROPECUÁRIA - EMBRAPA. Sistema brasileiro de classificação de solos. 2. ed. Rio de Janeiro: EMBRAPA Solos, 2006. 306p.

GALVAN, L.; OLIAS, M.; VILLARAN, R. F.;SANTOS, J. M. D. Aplicación del modelo hidrológico SWAT a la cuenca del rio Meca (Huelva, España). Geogaceta, v. 42, p. 6366, 2007.

GASSMAN, P. W.; JHA, M.; SECCHI, S.; ARNOLD, J. Initial calibration and validation of the SWAT model for the Upper Mississippi River Basin. In: DIFFUSE POLLUTION CONFERENCE, 2003, Dublin. Proceedings... Dublin: UCD, 2003. p. 1035-40.

GASSMAN, P. W.; REYES, M. R.; GREEN, C. H.; ARNOLD, J. G. The soil and water assessment tool: historical development, applications, and future research directions, Trans. ASAE, v. 50, n. 4, p. 1211- 1250, 2007.

GRIENSVEN, A. V. Sensitivity, auto-calibration, uncertainty and model evaluation in SWAT2005. Artigo técnico. 2005. Disponível em: <http://gisvrac.vrac.iastate.edu/icrw/download/PrintOut/SWAT2005_manual_sens_cal_unc.pdf.>. Acesso: 10 ago. 2009.

GUERREIRO, M. J.; ABREU, I.; BARROS, M. Variação anual e mensal de parâmetros meteorológicos na região do Algarve, Portugal. Revista da Faculdade de Ciência e Tecnologia, Porto, n. 2, p. 40-50, 2005. ISSN 1646-0499.

JHA, M.; GASSMAN, P. W.; SECCHI, S.; GU, R.; ARNOLD, J. Impact of watershed subdivision level on flows, sediment loads, and nutrient losses predicted by SWAT. Working Paper 02-WP 315. Center for Agricultural and Rural Development. Iowa State University. 2002. Disponível em: <http://econ2.econ.iastate.edu/research/webpapers/ paper_10054.pdf>. Acesso: maio 2010.

LINO, J. F. L. Análise da dinâmica hidrossedimentológica da bacia hidrográfica do Rio Preto (SC) com o modelo SWAT. 2009. 150f. Dissertação (Mestrado em Engenharia Ambiental) - Universidade Federal de Santa Catarina, Florianópolis, 2009. 
LOPES, N. H. Y. Análise da produção de água e sedimentos em microbacias experimentais com o modelo SWAT. 2008. 154f. Dissertação (Mestrado em engenharia Ambiental) - Departamento de Engenharia Sanitária e Ambiental, Universidade Federal de Santa Catarina, Florianópolis, 2008.

MACHADO, R. E. Simulação de escoamento e de produção de sedimentos em uma microbacia hidrográfica utilizando técnicas de modelagem e geoprocessamento. Tese (Doutorado em Irrigação e Drenagem) - Escola Superior de Agricultura Luiz de Queiroz, Universidade de São Paulo, Piracicaba, 2002.

MERMUT, A. R.; LUK, S. H.; ROMKENS, M. J. M.; POESEN, J. W. A. Soil loss by splash and wash during rainfall from two loss soils. Geoderma, v. 75, n. 3, p. 203-214, 1997.

MINOTI, R. T. Abordagens qualitativa e quantitativa de micro-bacias hidrográficas e áreas alagáveis de um compartimento do Médio Mogi-Superior/SP. 2006. 231f. Tese (Doutorado em Ciências da Engenharia Ambiental) - Escola de Engenharia de São Carlos, Universidade de São Paulo, São Carlos, 2006.

MORO, M. A utilização da interface SWAT-SIG no estudo da produção de sedimentos e do volume de escoamento superficial com simulação de cenários alternativos.2005. 100f. Dissertação (Mestrado em Agronomia) - Escola Superior de Agricultura Luiz de Queiroz, Universidade de São Paulo, Piracicaba, 2005.

MULETA, M. K.; NICKLOW, J. W. Sensitivity and uncertainty analysis coupled with automatic calibration for a distributed watershed model. Journal of Hydrology, v. 306, p. 127-145, 2005.

NEVES, F. F. Análise prospectiva das áreas de risco à erosão na microbacia hidrográfica do Rio Bonito (Descalvado - SP), potencialmente poluidoras por dejetos de granjas. 2005. 200f. Dissertação (Mestrado em Engenharia Ambiental) - Escola de Engenharia de São Carlos, Universidade de São Paulo, São Carlos, 2005.

NEITSCH, S. L.; ARNOLD, J. G.; KINIRY, J. R.; SRINIVASAN, R.; WILLIAMS, J. R. Soil and Water Assessment Tool Theoretical Documentation. Version 2005. Temple: Grassland, Soil and Water Research Laboratory, Agricultural Research Service, 2005. 476p.

NUNES, J. P.; PACHECO, N. R. Informação geográfica e modelação para a gestão de bacias hidrográficas: consequências das alterações climáticas para os processos hidrológicos e erosivos: potenciar a informação geográfica para a gestão ambiental. In: ESIG, 2004, Oeiras. Disponível em: <http://www.igeo.pt/servicos/cdi/biblioteca/publicacoesIGP/ ESIG_2004/p058.pdf>. Acesso: maio 2010.

OLIVEIRA, M. Q. C. Impacto de mudanças no uso do solo nas características hidrossedimentológicas da bacia hidrográfica do rio Joanes e sua repercussão na zona costeira. 1999. Dissertação (Mestrado em Geologia) - Universidade Federal da Bahia, Salvador, 1999.

OÑATE-VALDIVIESO, F. ; AGUILAR NARANJO, G. Aplicación del modelo SWAT para la estimación de caudales y sedimentos en la cuenca alta del rio Catamayo. In: CONGRESO LATINOAMERICANO DE MANEJO DE CUENCAS HIDROGRÁFICA, 3., 8 - 13 de junio 2003, Arequipa. Disponível em: < http://sig.utpl.edu.ec/sigutpl/Staftpro/hidrologia/swat_catamayo.pdf>. Acesso: maio 2010. 
PRADO, T. B. G. Evolução do uso das terras e produção de sedimentos na bacia hidrográfica do rio Jundiaí-Mirim. 2005. 72f. Dissertação (Mestrado em Agricultura Tropical e Subtropical) - Instituto Agronômico de Campinas, Campinas, 2005.

SANTHI, C.; SRINIVASAN, R.; ARNOLD, J. G.; WILLIAMS, J. R. A modeling approach to evaluate the impacts of water quality management plans implemented in a watershed in Texas. Environmental Modelling \& Software, v. 21, p. 1141-1157, 2006.

SANTOS, M. E. R. Características da forragem e produção de bovinos em pastagens de capim-braquiária diferidas. 2007. 100f. Dissertação (Mestrado em Zootecnia) Programa de Pós Graduação em Zootecnia, Universidade Federal de Viçosa, viçosa, MG, 2007.

SCHAEFER, C. E. R. G.; LIMA, H. N.; COSTA, O. V.; PASSOS, R. R. Levantamento de solos, paisagem e uso agrícola do Córrego São Bartolomeu, Viçosa, MG. Viçosa, MG: UFV, 1998. 46 p.

SCHUOL, J.; ABBASPOUR, K. C.; YANG, H.; SRINIVASAN, R.; ZEHNDER, A. J. B. Modeling blue and green water availability in Africa. Water Resour. Res., v. 44, 2008.

SMITH, M. Report on the expert consultations on revision of FAO methodologies for crop water requirements. Rome: FAO, 1991. 45p.

SRINIVASAN, R.; ARNOLD, J. G. Integration of the basin-scale water quality model with GIS. Water Resources Bulletin, v. 30, n. 03, p. 453-462, 1994.

SOUZA, M. N. Degradação e recuperação ambiental e desenvolvimento sustentável. 2004. 371f. Dissertação (Mestrado em Ciência Florestal) - Universidade Federal de Viçosa, Viçosa, MG, 2004.

TRIPATHI, M. P.; PANDA, R. K.; RAGHUWANSHI, N. S. Identification and prioritization of critical sub-watersheds for soil conservation management using the SWAT model. Biosystems Engineering, v. 85, n. 3, p. 365-379, 2003.

TUCCI, C. E. M. Modelos hidrológicos. Porto Alegre: UFRGS, Associação Brasileira de Recursos Hídricos, 1998. 943p.

VAHOS M., J. D. Generación de modelos digitales hidrológicos y de pérdida de suelo y su comparación con métodos tradicionales en la Cuenca del Río Claro (Suroeste Antioqueño). 2003. 106f. Trabajo (Grado Ingeniero Florestal) - Facultad de Ciencias Agropecuárias, Universidad Nacional de Colômbia, Bogotá, 2003.

VILELA, M. Uso de diferentes métodos de retificação geométrica e classificação digital de uma imagem TM/LANSAT - 5. 1998. 118f. Dissertação (Mestrado em Engenharia Florestal) - Depto. de Engenharia Florestal, Universidade Federal de Viçosa, viçosa, MG, 1998.

VILELA, M. F; ROMANOVZKI, Z. O uso do solo e a conservação da vazão dos cursos d’água na bacia do ribeirão São Bartolomeu, Viçosa - MG. In: SIMPÓSIO BRASILEIRO DE SENSORIAMENTO REMOTO, 10. 21-26 abril 2001, Foz do Iguaçu. Anais... São José dos Campos: INPE, 2001. p. 699-706.

ZHANG, X.; SRINIVASAN, R.; VAN LIEW, M. Multi site calibration of the SWAT model for hydrologic modeling. American Society of Agricultural and Biological Engineers, v. 51, n. 6, p. 2039-2049, 2008. 